\title{
Unsteady Lift Suppression with a Robust Closed Loop Controller
}

\author{
David Williams ${ }^{1}$, Wesley Kerstens ${ }^{1}$, Jens Pfeiffer ${ }^{2}$, Rudibert King ${ }^{2}$, Tim \\ Colonius $^{3}$ \\ ${ }^{1}$ Fluid Dynamics Research Center, Illinois Institute of Technology, Chicago \\ IL 60616 USA \\ williamsd@iit.edu \\ ${ }^{2}$ Measurement and Control Group, Technische Universität Berlin, Harden- \\ bergstr. 36a, 10623 Berlin, Germany \\ Rudibert.king@tu-berlin.de \\ ${ }^{3}$ Mechanical Engineering Department, California Institute of Technology, \\ Pasadena CA 91125 \\ colonius@caltech.edu
}

\begin{abstract}
Summary
The ability to control lift in unsteady flows using active flow control is examined experimentally with a three-dimensional, low-aspect-ratio wing and pulsedblowing actuators as the test article. An unsteady flow wind tunnel is used to generate step-like and harmonic oscillations in flow speed and the corresponding fluctuating lift force on the wing. A 'black box' model of the wing response to actuation is obtained using conventional system identification techniques. A robust $\mathrm{H}_{\infty}$ controller is designed with a mixed sensitivity loop-shaping technique, whose objective was to maintain a constant lift in the unsteady flow. The controller is shown to be capable of significant reductions in lift fluctuations given step, harmonic and random input disturbance conditions.
\end{abstract}

\section{Introduction}

Dynamic control of lift is a requirement for maneuvering aircraft, whether the control is done with conventional control surfaces or with modern active flow control actuators. Modern active flow control (AFC) offers the advantage of lift en- 
hancement and the delay of stall, but the demonstrations of AFC have been done almost exclusively under steady state conditions, i.e., constant flow speed and constant (periodic) actuation, e.g. Neuberger \& Wygnanski [1] and Seifert, et al. [2,3]. Enabling AFC techniques to be useful for aircraft maneuvering requires an understanding of the performance of both the AFC actuator and the flow over the flight vehicle in unsteady flow environments.

The challenge in using AFC to enhance aircraft maneuverability comes from the effects of unsteady aerodynamics and the substantial time delays in the flow response to actuation. A quasi-steady control approach only works at very slow maneuvering speeds, because the static response maps become highly inaccurate in dynamic situations for both the aerodynamic loads and the response to actuation. For example, the time delays in the lift response to actuator transients was found to be quite long in the experiments by Amitay \& Glezer [4,5] on a 2D wing, Darabi \& Wygnanski [6,7] on a 2D wing flap, and Williams, et al.[8] on a 3D wing. Relaxation times of the flow from the actuator's initial transient to the final steady state were found to be on the order of $\mathrm{O}\left(5-10 \mathrm{t}^{+}\right)$, where $\mathrm{t}^{+}$is the convective time scale, which is time normalized by the external flow speed, $U$, and the chord length of the wing or flap model, c. This means that a wing will travel $5-10$ chord lengths before its response to a change in actuator settings is complete.

A benchmark experiment was designed to explore the challenges of using AFC in an unsteady flow [9]. The basic idea was to superpose flow speed oscillations on a mean flow to create unsteady lift oscillations from a wing, simulating the effects of a longitudinal gusting flow. A phase-cancellation control system was designed around pulsed-blowing AFC actuators to modulate the lift coefficient, so that the lift oscillations would be suppressed. The first attempts by Williams, et al. [9] at suppressing lift fluctuations in an oscillating flow used a controller based on a quasi-steady model of the aerodynamics and actuator response. The actuators used a pulse-width-modulation approach to modify the lift coefficient. The approach was effective at suppressing harmonic disturbances at the fundamental frequency of $0.2 \mathrm{~Hz}$, but introduced a large amount of noise at higher harmonics. The quasi-steady models for the actuator and aerodynamics were the limiting factor in the design.

An improved version of the phase-cancellation approach was demonstrated by Williams, et al. [10]. The improvement accounted for the time delays associated with the unsteady aerodynamics and the actuation. Experimental measurements of the time delays at different flow oscillation frequencies were obtained, and a phase cancelling feed forward controller was designed to suppress lift oscillations at a fixed frequency $(1 \mathrm{~Hz})$. Good suppression of the lift oscillations (typically $\sim 10-$ $12 \mathrm{~dB}$ ) was demonstrated at the design frequency, but only at the design point. If the disturbance frequency or amplitude was changed, then the controller would quickly lose effectiveness. Despite the limited bandwidth, the concept of suppressing unsteady lift oscillations with a controller that was correctly tuned for the unsteady aerodynamic effects and the time delays associated with actuation was proved. 
Broader bandwidth control is necessary to achieve enhanced maneuverability and gust suppression in actual flight conditions. The objective of the work described in this paper is to use the techniques of modern system identification and control theory to increase the bandwidth of the controller. In particular, the approach developed by Henning, et al. [11] provides a template for designing a modern controller capable of suppressing lift oscillations in the unsteady flow problem. Experiments were conducted at Illinois Institute of Technology and the controllers were designed at Technische Universität Berlin. The methodology and results of the joint effort are described below.

The experimental setup and flow configuration are described in Sec.2. Openloop forcing results and the static response to actuation are discussed in Sec. 3. The closed-loop controller design and experimental results are provided in Sec.4. Finally, the conclusions of the experiment are presented in Sec. 5.

\section{Flow Configuration}

The model used in this study was a low-aspect-ratio, low Reynolds number wing mounted on a vertical sting in a wind tunnel. The wing was fixed at $\alpha=20 \mathrm{deg}$, which without flow control was in a stalled state. The wind tunnel was equipped with a computer controlled shutter mechanism that changes the resistance to the flow in the wind tunnel. Different waveforms of velocity disturbances can be created, but for this work step-type and harmonic oscillations were used. Oscillation amplitudes up to 10 percent of the mean speed and frequencies up to $3 \mathrm{~Hz}$ were achievable.

\subsection{Experimental Setup}

The test article for the experiment was a semi-circular planform wing. The semi-circular planform was chosen because earlier experiments documented the ability of pulsed-blowing actuators to stabilize and modulate the strength of the leading edge vortex and lift, Williams, et al.[12]. The model was constructed from Duraform ${ }^{\mathrm{TM}}$ nylon with a selective laser sintering rapid prototyping system. The 3D rapid prototyping system allowed the micro-valve actuators to be embedded inside the model, close to the leading edge. The midspan chord $=0.203 \mathrm{~m}$, and the $\mathrm{span}=.406 \mathrm{~m}$. The leading-edge was rounded with a 5:1 elliptic shape, and the thickness to chord ratio was $t / c=0.069$. The model chord Reynolds numbers ranged from $\operatorname{Re}_{\mathrm{c}}=47,000$ to 109,000 .

The experiments were conducted in the Andrew Fejer Unsteady Flow wind tunnel. The wind tunnel is a closed-return type powered by a $40 \mathrm{HP}$ motor with vector drive controller. The maximum speed of the wind tunnel is $30 \mathrm{~m} / \mathrm{s}$, but ranged between $3 \mathrm{~m} / \mathrm{s}$ and $9 \mathrm{~m} / \mathrm{s}$ for the data presented here. At $3 \mathrm{~m} / \mathrm{s}$ freestream 
speed the turbulence level is 0.6 percent over the frequency band from $0.1 \mathrm{~Hz}$ to $30 \mathrm{~Hz}$. The test section dimensions are $0.6 \mathrm{~m}$ by $0.6 \mathrm{~m}$ by $3.1 \mathrm{~m}$ long.
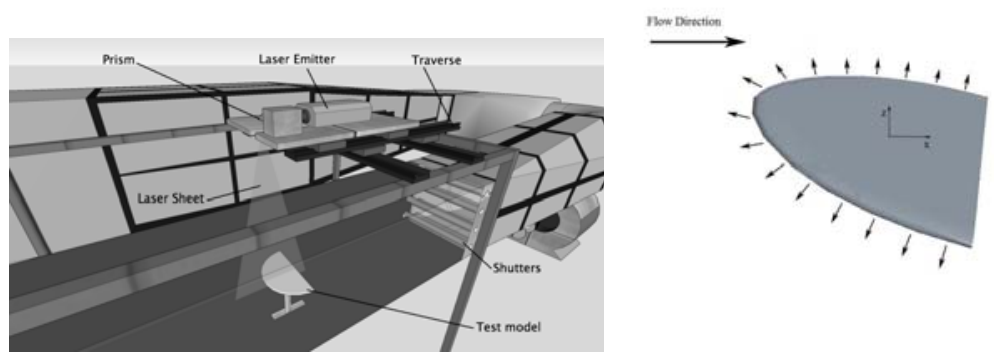

Figure 1 - Drawing of wind tunnel test section showing the model and shutter arrangements. The actuator orientation along the leading edge is shown on the right.

The coordinate system was defined relative to the force and moment transducer located inside the model. The $\mathrm{x}$-axis is aligned with the streamwise direction, the $y$-axis is in the spanwise direction, and the z-axis is in the vertical direction relative to the flow in the wind tunnel. The forces and moments about the three principle axes were acquired with a 6-component balance (ATI, Inc. model Nano-17).

The angle of attack of the wing was fixed at $\alpha=20 \mathrm{deg}$., which corresponds to an area ratio blockage of 6 percent. The unsteady nature of the experiments makes it unclear how blockage corrections should be applied; therefore, no blockage corrections were made to the data.

The actuation system was a pulsed-blowing type with variable pressure amplitude. The actuator pressure amplitude was controlled by a Fairchild TA6000 pneumatic transducer, which had an approximate bandwidth of $2 \mathrm{~Hz}$. The flow from the pneumatic transducer supplied a plenum inside the wing. The 16 micro valve (Lee, Inc. model LHDA00102) actuators were positioned radially outward along the leading edge of the wing. In typical open-loop actuation the valves are pulsed continuously at $29 \mathrm{~Hz}(\mathrm{St}=1.2$ at $\mathrm{U}=5 \mathrm{~m} / \mathrm{s})$.

\section{Open-Loop Control}

Maps of the steady state lift response to actuation were obtained as a first step in the controller design. The steady lift (with and without actuation) and the dependence on actuator pressure are described in this section.

\subsection{Baseline Flow with and without actuation}


The smoke wire technique was used to visualize the flow over the midspan of the wing. The baseline flow without actuation is shown in Fig. $2 \mathrm{a}$ and the open-loop, continuous forcing case is shown in Fig. 2b. Both cases correspond to a steady freestream speed at $5 \mathrm{~m} / \mathrm{s}$.
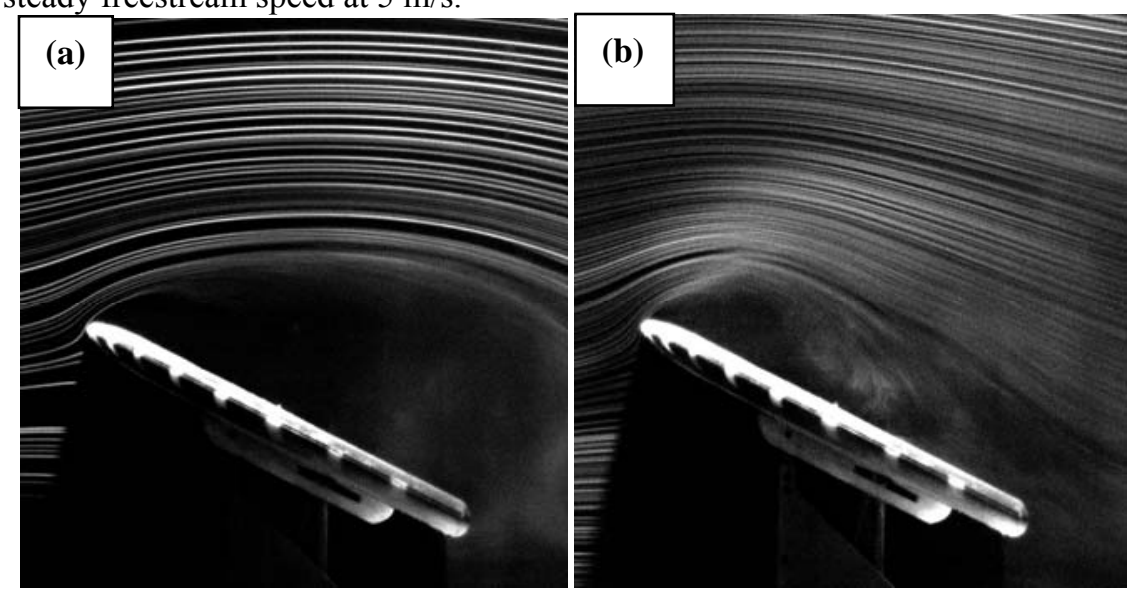

Figure 2. Smoke wire visualization of flow over the mid-span of the wing at $\mathrm{U}=5 \mathrm{~m} / \mathrm{s}$. (a) no forcing. (b) continuous pulsed-jet actuation at $29 \mathrm{~Hz}, 5 \mathrm{psig}$

The smoke visualization photograph in Fig. $2 \mathrm{~b}$ shows the reattached flow pattern that produces a high lift state when the actuators are running continuously at $29 \mathrm{~Hz}$.

The effect of continuous actuation can be seen in the lift curves shown in Fig.3. for a flow speed of $5 \mathrm{~m} / \mathrm{s}$. The solid black line shows the lift dependence on angle of attack, $\alpha$, without actuation. The wing stalls at $\alpha=14$ degrees, and at $\alpha=20$ degrees the lift decreases to $0.8 \mathrm{~N}$, which is the lower lift limit. The upper lift limit is obtained with the actuators pulsing continuously at $25 \mathrm{~Hz}$. The stall is delayed, and the upper lift value $(\mathrm{L}=1.2 \mathrm{~N})$ is reached. The basic control strategy is to fix the wing at $\alpha=20$ degrees, and to change the lift force between the upper and lower limits by changing the supply pressure to the actuators. 


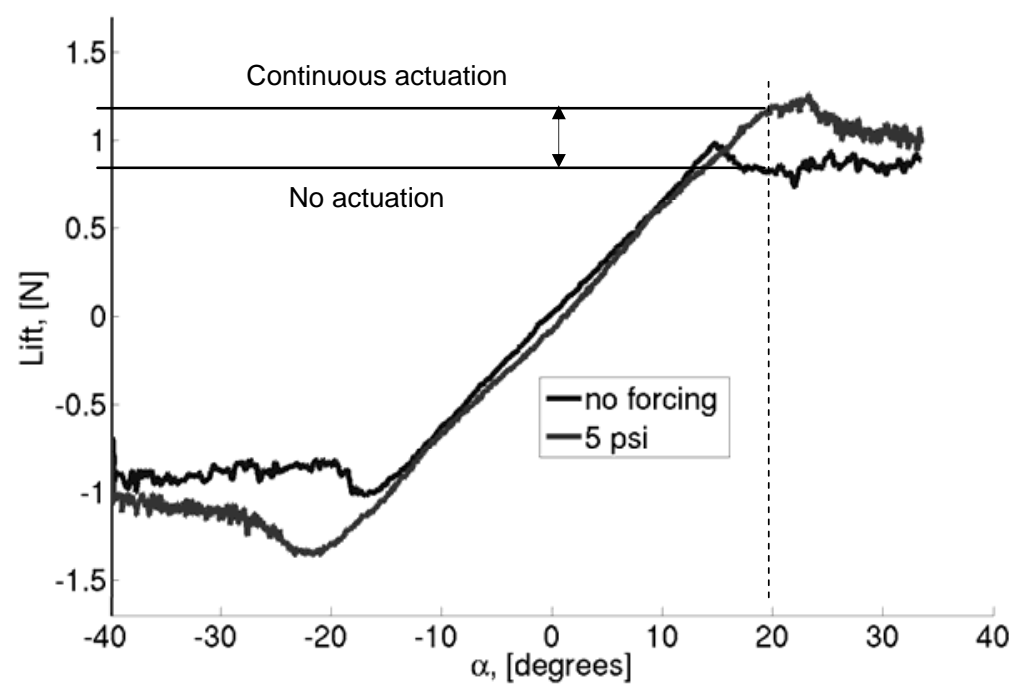

Figure 3. Lift curve dependence on actuation at $U=5 \mathrm{~m} / \mathrm{s}, \operatorname{Re}_{\mathrm{c}}=78,000$. The black line is the baseline case without forcing. The gray line is the lift with continuous actuation.

\subsection{Description of Actuated Flow - Static Map}

The static lift response of the wing to continuously pulsed (steady-state) actuation is shown in Fig. 4. The data were found to scale well when the lift coefficient was plotted against the square-root of the actuator supply pressure coefficient. The supply pressure coefficient is defined as $C_{p j}=\frac{p_{j e t}-p_{\infty}}{0.5 \rho U^{2}}$, where $\mathrm{p}_{\text {jet }}$ is the actuator supply pressure and $\mathrm{p}_{\infty}$ is the freestream static pressure. The square-root dependence on $\mathrm{C}_{\mathrm{pj}}$ is modeled as a static input nonlinearity in the plant model. Details are given in Sec. 4.1. 


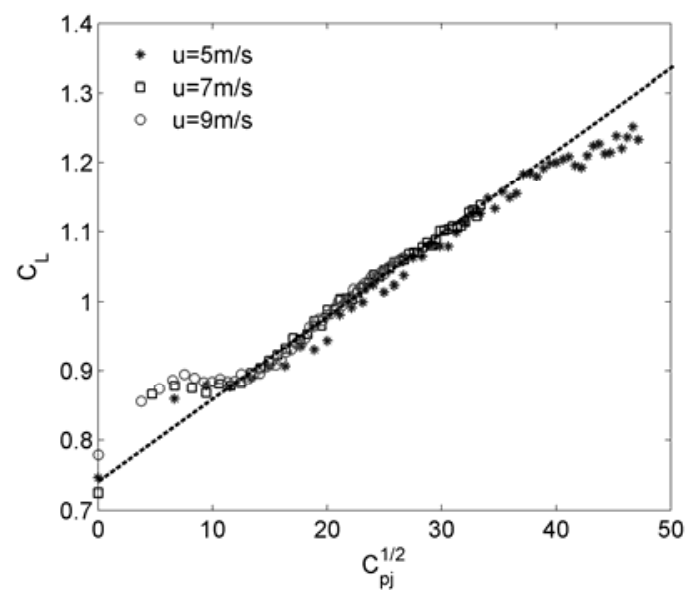

Figure 4. Lift coefficient dependence on the actuator supply pressure. The angle of attack is fixed at $\alpha=20 \mathrm{deg}$. The actuators are pulsed continuously at $29 \mathrm{~Hz}$.

\section{Feedback Control}

The controller and the design procedure used by Henning, et al. [11] provided a template for the design of the controller used in the current experiment. A modelbased robust controller was designed using the mixed-sensitivity $\mathrm{H}_{\infty}$ control method [14]. The control architecture, system identification, and performance in unsteady flow experiments are described in the following.

\subsection{Robust Control}

The schematic in Fig. 5 shows the control loop architecture used to regulate the lift of the wing. The steady-state map obtained from open-loop experiments plotted in Fig. 4 reveals an approximately linear relationship between the lift coefficient $\mathrm{C}_{\mathrm{L}}$ and the square root of the jet pressure coefficient $\mathrm{C}_{\mathrm{pj}}$. Hence the plant with input $\mathrm{u}=\mathrm{C}_{\mathrm{pj}}$ and output $\mathrm{y}=\mathrm{C}_{\mathrm{L}}$ can be modeled as a nonlinear system consisting of a linear dynamic part $\mathrm{G}_{\mathrm{p}}(\mathrm{s})$ and a static input nonlinearity $\mathrm{f}(\mathrm{u})=\mathrm{C}_{\mathrm{pj}}{ }^{1 / 2}$. Its inverse is used as a pre-compensator $\mathrm{f}\left(\mathrm{u}^{*}\right)^{-1}=\mathrm{u}^{* 2}$, resulting in a plant which is linear with respect to $u^{*}$ and $y$. The plant transfer function $G_{p}(s)$ is a 'black-box' model obtained from a system identification based on experimental data.

Since the plant is modeled with respect to the nondimensional coefficients $C_{p j}$ and $\mathrm{C}_{\mathrm{L}}$, the desired lift $\mathrm{L}_{\mathrm{ref}}$ has to be converted to a reference value for the lift co- 
efficient $\mathrm{r}=\mathrm{C}_{\mathrm{L} \text {,ref }}$ based on the measured free-stream dynamic pressure $\mathrm{q}$ and the wing surface $\mathrm{S}$.

A model-based dynamic feed forward controller $K_{f f}(s)=F(s) G_{n}(s)^{-1}$ is used to speed up the reference tracking. Herein $\mathrm{G}_{\mathrm{n}}(\mathrm{s})^{-1}$ denotes the inverse of the linear transfer function of a nominal plant model and F(s) denotes a PT1 reference filter needed to obtain a causal transfer function for $\mathrm{K}_{\mathrm{ff}}(\mathrm{s})$. A robust $\mathrm{H}_{\infty}$ controller $\mathrm{K}(\mathrm{s})$ designed by loop-shaping compensates for tracking errors caused by disturbances and modeling errors. In order to account for actuator saturation the control loop is augmented by a dynamic Anti-windup compensator based on a method suggested by Park [13]. It is not shown in Fig. 5 for the sake of conciseness.

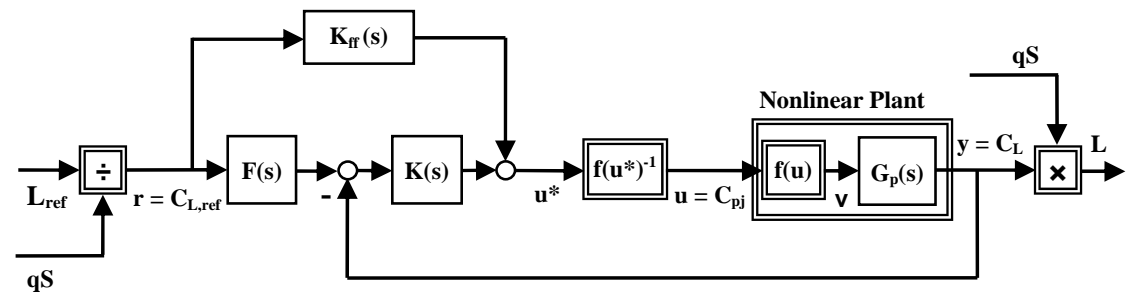

Figure 5. Control loop architecture used for suppression of the oscillating lift.

\subsubsection{Identification of the plant model}

The lift coefficient response to the actuator shown in Fig. 4 is only valid for the static response. A dynamic model $\mathrm{G}_{\mathrm{p}}(\mathrm{s})$ of the plant is needed for the controller, which can be obtained from experimental data using conventional system identification methods. In this case the compensated input $\mathrm{u}^{*}(\mathrm{t})$ corresponding to the square root supply pressure coefficient $\left(\mathrm{C}_{\mathrm{pj}}{ }^{1 / 2}\right)$ provided to the actuators is varied as a pseudo-random binary signal (PRBS). The output is the measured lift coefficient. Based on the output response to different amplitudes of the input signals at three different flow speeds $U=5 \mathrm{~m} / \mathrm{s}, U=7 \mathrm{~m} / \mathrm{s}$ and $U=9 \mathrm{~m} / \mathrm{s}$ a family of 33 'black box' models is identified using the Prediction-Error-Method. A comparison between the measured response and the simulated output for one identified model for a sample of the pseudo-random input data at one supply pressure condition is shown in Fig. 6. 

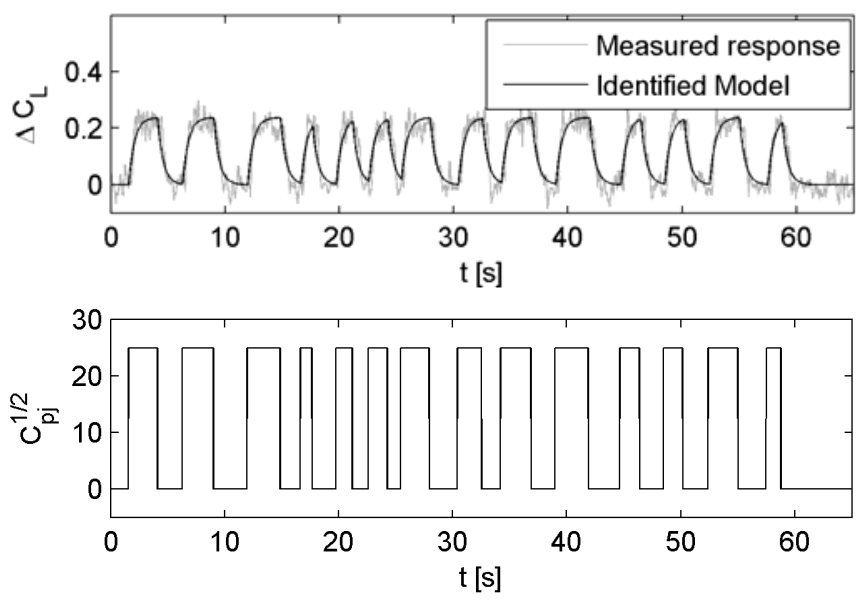

Figure 6. Example of pseudo-random input data used to obtain a 'black box' model of the wing's lift response.

A PT1 structure for the model identification yields a reasonable fit of the measured plant output and was therefore used in the identification and control design process. However, recent experiments not shown here suggest that considering a time delay or a higher model order could improve the data fit and control performance.

A nominal model $\mathrm{G}_{\mathrm{n}}(\mathrm{s})$ is obtained by calculating the mean values of the parameters from all models. The Bode plot of all 33 models and the nominal model shown in figure 7 reveals a small variation in the frequency response of the models. The deviation from the nominal model's frequency response is described by a multiplicative uncertainty

$$
\Pi_{I}: G_{p}(s)=G_{n}(s)\left(1+w_{I}(s) \Delta_{I}(s)\right), \quad\left|\Delta_{I}(j \omega)\right| \leq 1, \quad \forall \omega,
$$

wherein $\Delta_{\mathrm{I}}(\mathrm{s})$ denotes a normalized uncertainty with a frequency dependent weight $\mathrm{w}_{\mathrm{I}}(\mathrm{s})$ comprising all identified transfer functions. 

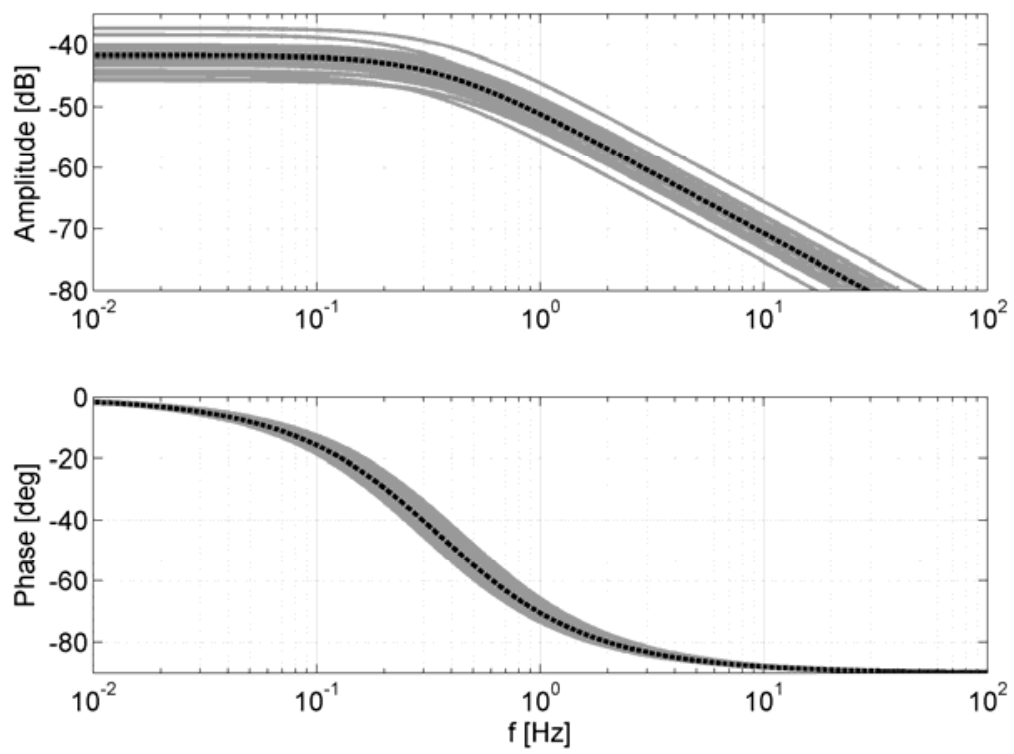

Figure. 7. Bode plots of the models identified from experiment (gray) and nominal model (dashed)

\subsubsection{Mixed sensitivity method of controller design}

The mixed-sensitivity method of loop-shaping is used to tune the controller performance, so that robust stability and performance are guaranteed for all models identified over the range of flow speeds from $5 \mathrm{~m} / \mathrm{s}$ to $9 \mathrm{~m} / \mathrm{s}$. In this approach, the loop transfer functions for the sensitivity $S=\left(1-G_{n} K\right)^{-1}$, the complementary sensitivity $\mathrm{T}=1-\mathrm{S}$, and $\mathrm{KS}$ as a measure for the magnitude of the input signals are weighted with $\mathrm{w}_{\mathrm{P}}(\mathrm{s}), \mathrm{w}_{\mathrm{T}}(\mathrm{s})$ and $\mathrm{w}_{\mathrm{u}}(\mathrm{s})$, respectively, to combine several specifications for the controller to be synthesized. Here, $1 /\left|\mathrm{w}_{\mathrm{P}}(\mathrm{s})\right|$ is used to put a lower bound on the bandwidth to suppress disturbances at low frequencies. The weight $\mathrm{w}_{\mathrm{T}}(\mathrm{s})$ ensures that the magnitude of $\mathrm{T}$ rolls off fast enough at high frequencies to avoid transmission of measurement noise. Furthermore, it is chosen such that

$$
|T(j \omega)|<1 /\left|w_{I}(j \omega)\right|, \forall \omega,
$$

in order to guarantee robust stability [14]. Since the magnitude of the uncertainty $\mathrm{W}_{\mathrm{I}}$ is smaller than unity at all frequencies $\omega$, no limitations on the control design arise from the uncertainty. Finally, $1 /\left|\mathrm{w}_{\mathrm{u}}(\mathrm{s})\right|$ places an upper bound on the magnitude of the control effort. The Bode plots of these transfer functions are shown in Fig. 8. In order to obtain the controller a cost functional

$$
\min _{K}\|N(K(s))\|_{\infty} \text {, with } N=\left[\begin{array}{lll}
w_{P} S & w_{T} T & w_{u} K S
\end{array}\right]^{\mathrm{T}},
$$

has to be minimized, wherein $\mathrm{K}(\mathrm{s})$ denotes the optimal controller [14]. 


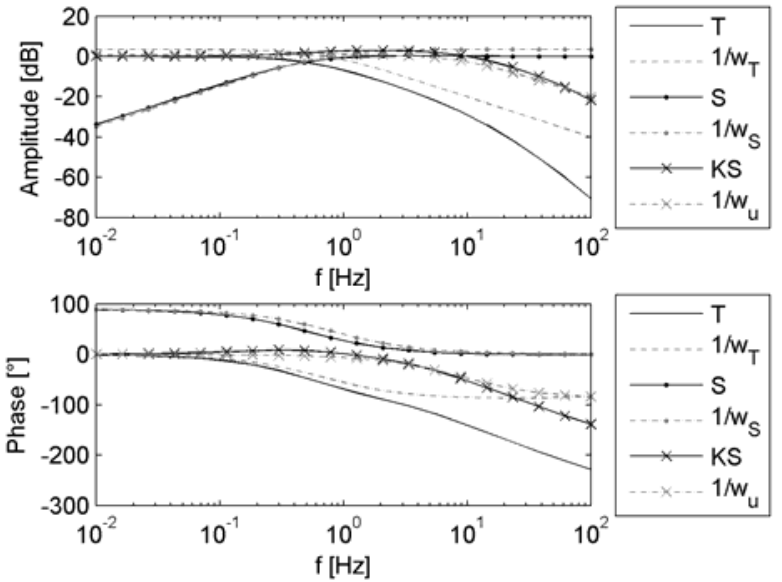

Figure 8 . Bode plots of the shaped transfer functions used in the design of the controller. Refer to text for details.

\subsection{Controller performance in experiments}

Step input changes in the freestream speed were used to test the controller. The wing was subjected to a periodic step decrease in velocity from approximately 7.1 $\mathrm{m} / \mathrm{s}$ to $6.3 \mathrm{~m} / \mathrm{s}$, while the controller attempted to maintain a constant reference lift value of $1.4 \mathrm{~N}$. Figure 9 shows the phase-averaged lift response over 50 cycles of the controlled case along with the uncontrolled lift. Oscillations in lift occur when the flow speed transient occurs, but these oscillations are damped over time. The controller is successful in maintaining a constant lift near the reference value 1.4 $\pm 0.05 \mathrm{~N}$.
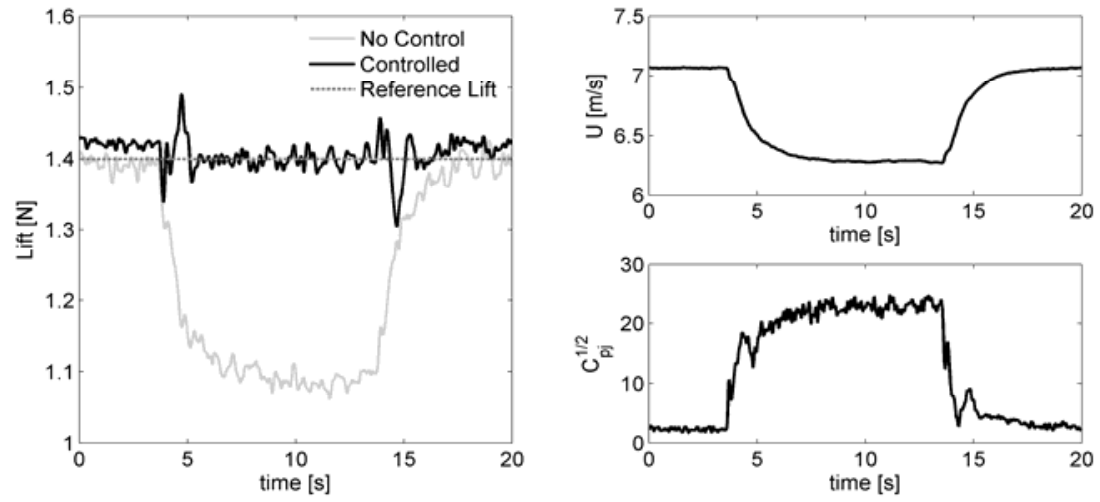

Figure 9. Step response of lift at $U=7 \mathrm{~m} / \mathrm{s}$ with and without control (left). Phase averaged velocity signal (top right) and control input signal, $C_{p j}^{1 / 2}$, (bottom right.) 
The wing was also subjected to a $0.2 \mathrm{~Hz}$ sinusoidal velocity variation with a mean flow speed of $6.7 \mathrm{~m} / \mathrm{s}$ and amplitude of $0.25 \mathrm{~m} / \mathrm{s}$. Again the controller attempts to maintain a constant lift. Figure 10 shows the power spectral density of the controlled and uncontrolled fluctuating lift.
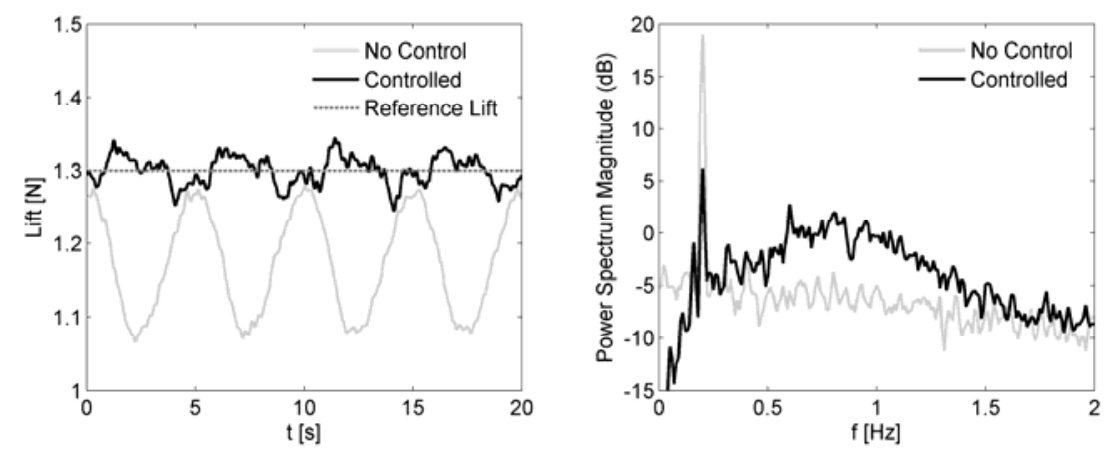

Figure. 10. Comparison of fluctuating lift signal with and without control (left), and the power spectra of both signals (right). A $13 \mathrm{~dB}$ suppression of the lift oscillation is obtained at the fundamental frequency with only a slight increase at the harmonic.

The ability of the controller to maintain a constant lift given a pseudo-random input velocity signal is shown in Fig. 11. Without control, the lift varies from 0.9 $\mathrm{N}$ to $1.4 \mathrm{~N}$. With the controller activated, the lift fluctuation amplitude is significantly reduced. The root mean square value of the fluctuating lift is reduced from $0.14 \mathrm{~N}$ without control to $0.028 \mathrm{~N}$ with control. The ability to control random inputs is an important step toward developing gust suppression controllers. 

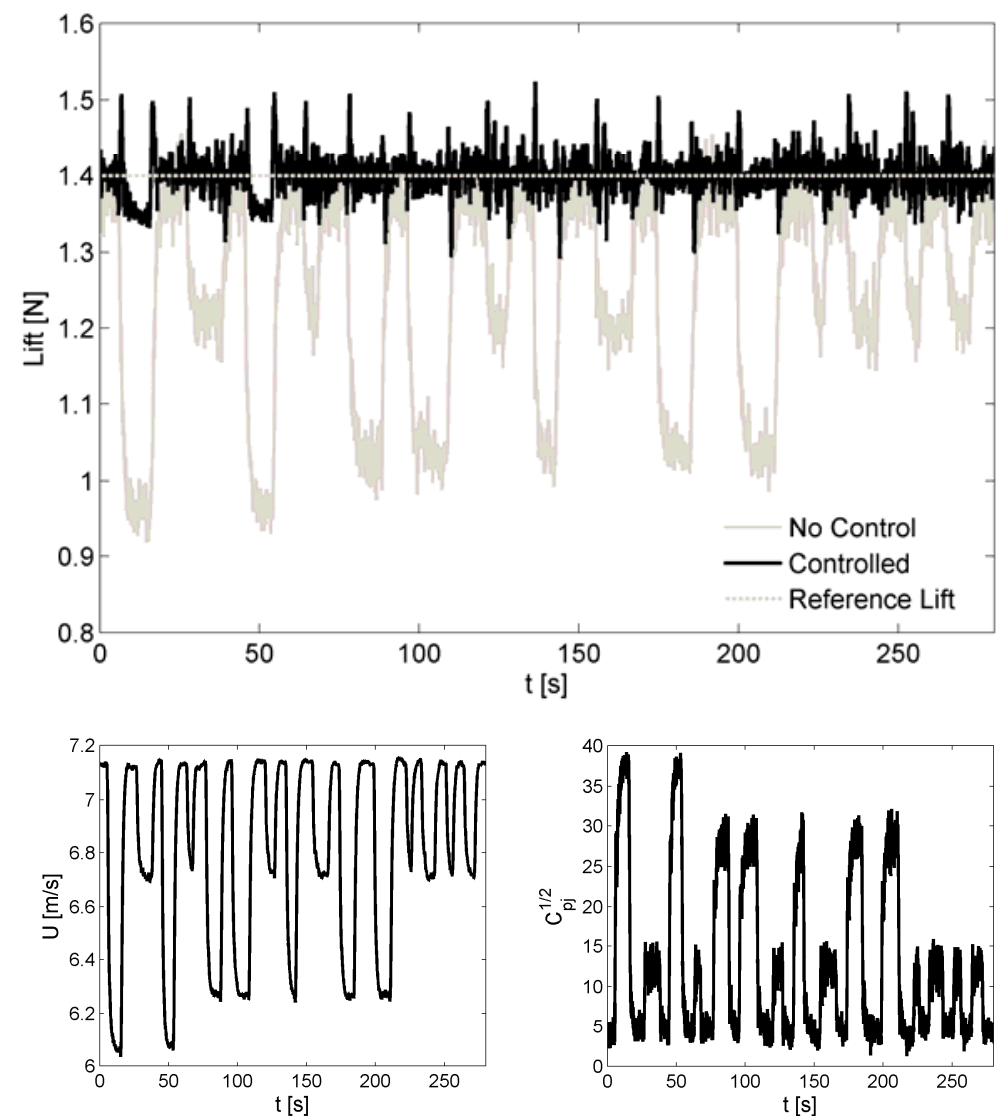

Figure 11. Randomized input velocity. The lift response with and without control (top) shows suppression of the lift fluctuations. The corresponding freestream velocity signal (bottom left) and the control input signal $C_{p j}^{1 / 2}$ (bottom right) are shown.

\section{Conclusions}

A robust controller was designed with the objective of suppressing freestream flow oscillations over a wide range of unsteady flow conditions in benchmark experiment with a 3D wing. Previous controllers used at IIT were only effective at their design point or under quasi-steady conditions. The controller design procedure used by Henning, et al. [11] for controlling bluff body drag, also proved to be effective for constructing a controller capable of modulating the lift in an unsteady 
flow situation. A family of 'black box' models was identified using experimental measurements, and a robust $\mathrm{H}_{\infty}$ controller coupled with dynamic feed forward control worked well. The controller presented in this paper was shown to be effective in compensating for step, harmonic, and random input changes in flow speed.

\section{Acknowledgements}

The support for this work by the U.S. Air Force Office of Scientific Research MURI (FA9550-05-0369) with program manager Dr. Fariba Fahroo is gratefully appreciated. David Williams gratefully acknowledges the partial support of the Alexander von Humboldt foundation. We also acknowledge the support from the Illinois NASA Space Grant Consortium for Wesley Kerstens.

\section{References}

[1] Neuberger, D. and Wygnanski, I.: The use of a vibrating ribbon to delay separation on two-dimensional airfoils. Proc. of Air Force Academy Workshop in Unsteady Separated Flow, F.J. Seiler, ed., Report TR-88-0004 (1987)

[2] Seifert, A., Bachar, T., Koss, D., Shepshelovic, M., and Wygnanski, I.: Oscillatory blowing: a tool to delay boundary layer separation. AIAA J. 34 (1993) 2052-2060

[3] Seifert, A., Darabi, A., and Wygnanski,I.: Delay of airfoil stall by periodic excitation. J. Aircraft 33 (1996) 691-698

[4] Amitay, M., Glezer, A.: "Controlled Transients of Flow Reattachment over Stalled Airfoils. Int. J. of Heat Transfer and Fluid Flow 23 (2002) 690-699

[5] Amitay, M., and Glezer, A.: Flow Transients Induced on a 2D Airfoil by PulseModulated Actuation. Experiments in Fluids 240 (2006) 329-331

[6] Darabi, A., and Wygnanski, I.: Active management of naturally separated flow over a solid surface. Part 1. The forced reattachment process. J. Fluid Mech. 510 (2004) 105129

[7] Darabi, A., and Wygnanski, I.: Active management of naturally separated flow over a solid surface. Part 2. The separation process. J. Fluid Mech. 510 (2004) 131-144

[8] Williams, D.R., Tadmor, G., Colonius, T., Kerstens, W., Quach, V., and Buntain, S. The lift response of a stalled wing to pulsatile disturbances. AIAA J. 47 (2009)

[9] Williams, D., Collins, Tadmor, G., and Colonius, T.: Control of a Semi-Circular Planform Wing in a "Gusting" Unsteady Freestream Flow: I-Experimental Issues. AIAA Paper 2008-3976 (2008)

[10] Williams, D.R., Quach, V., Kerstens, W., Buntain, S., Tadmor, G., Rowley, C., Colonius, T.: Low-Reynolds Number Wing Response to an Oscillating Freestream with and without Feed Forward Control. AIAA Paper 2009-143 (2009)

[11] Henning, L., Pastoor, M., King, R., Noack, B., Tadmor, G.: Feedback Control Applied to the Bluff Body Wake. In: R. King (Ed.):Active Flow Control, NNFM 95, Springer, (2007) 369-390

[12] Williams, D., Collins, J., Jankhot, C., Colonius, T., and Tadmor, G.: Control of Flow Structure on a Semi-Circular Planform Wing. AIAA Paper 2008-597 (2008) 
[13] Park, J.-K. \& Choi, C.-H.: Dynamic compensation method for multivariable control systems with saturating actuators. IEEE Transactions on Automatic Control 40 (1995) $1635-1640$

[14] Skogestad, S., Postlethwaite, I.: Multivariable feedback control - Analysis and design. John Wiley \& Sons, Chichester, England (1996) 